Research Article

\title{
Multifunctional Fluorocarbon-conjugated Nano- particles of Varied Morphologies to Enhance Diagnostic Effects in Breast Cancer
}

\author{
Melissa Ronni Laughter ${ }^{1+}$, Anna Laura Nelson ${ }^{1+}$, Maria Bortot ${ }^{1}$, Brisa Pena ${ }^{1}$, Bolin Liu ${ }^{2}$, Daewon Park ${ }^{1}$ \\ ${ }^{1}$ Department of Bioengineering, University of Colorado Denver Anschutz Medical Campus, Aurora, CO 80045 USA. \\ ${ }^{2}$ Department of Genetics, Stanley S. Scott Cancer Center, Louisiana State University Health Sciences Center, New Orleans, LA 70112 \\ USA. \\ + These authors contributed equally to this work. \\ Corresponding author. E-mail: daewon.park@ucdenver.edu
}

Received:Sep. 10, 2017; Accepted: Sep. 20, 2017; Published: Sep. 27, 2017

Citation: Melissa Ronni Laughter, Anna Laura Nelson, Maria Bortot, Brisa Pena, Bolin Liu, and Daewon Park, Multifunctional Fluorocarbonconjugated Nanoparticles of Varied Morphologies to Enhance Diagnostic Effects in Breast Cancer. Nano Biomed. Eng., 202I, I3(I): 52-6I.

DOI: 10.5101/nbe.v13i1.p52-61.

\begin{abstract}
A multifunctional trastuzumab-nanoparticle-fluorocarbon system was developed to maximize the diagnostic effects in human epidermal growth factor receptor 2 (HER2)-positive breast cancer. The mesoporous silica nanoparticle shape (e.g. amorphous, spherical, and tubular) was altered to optimize the ultrasound contrast potential. Fluorocarbon conjugated mesoporous silica nanoparticles produced higher mean pixel intensities. At lower non-toxic concentrations, tubular shaped nanoparticles produced a higher mean pixel intensity compared to amorphous and spherical particles. All systems displayed a clear binding preference towards HER2-positive breast cancer cells. Increased incubation times and conjugation of fluorocarbon to mesoporous nanoparticles increased binding preference to HER2-positive breast cancer cells. The highest binding affinity was seen with tubular shaped nanoparticles as compared to amorphous and spherical particles. The trastuzumab-nanoparticlefluorocarbon system of each morphology displayed functionality of enhancing contrast in ultrasound.
\end{abstract}

Keywords: Mesoporous silica nanoparticles, Ultrasound, Contrast agent, Breast cancer, Diagnostic

\section{Introduction}

Breast cancer continues to be the most prevalent carcinoma amongst women in the United States, affecting over one million women each year [1]. Approximately $20 \%$ of these breast cancers possess amplified and/or overexpression of human epidermal growth factor receptor 2 (HER2) indicating a highly aggressive tumor [2,3]. For these patients, the standard of care includes the administration of the drug trastuzumab (Herceptin, Hoffmann-La Roche, Basel, Switzerland), an anti-HER2 monoclonal antibody that targets the HER2 transmembrane receptor leading to cancer cell death [4]. Although this drug represents a significant breakthrough in the treatment of resistant breast cancers, there lacks a reliable and cost-effective method to track the efficacy and progression of the treatment. Earlier and more sensitive assessment of trastuzumab effects on HER2 positive (HER2+) breast cancer can avoid overtreatment and unnecessary costs.

Ultrasound is an extremely appealing imaging technique for the non-invasive assessment of anti-cancer therapy [5, 6]. Ultrasound is cost-effective, has a high safety profile, and has the ability to provide real-time 
images that can be easily implemented into outpatient clinic visits [7, 8]. However, ultrasound has been known to have several limitations, such as image distortion, low resolution and low contrast, making it somewhat unreliable for detecting exceedingly small cancers and cancer treatment progression [8, 9]. These limitations could potentially be alleviated by increasing the contrast of the targeted tissue [10]. For this reason, researchers have turned to targeted drug therapy combined with some form of contrast enhancement in order to track the progression of tumor contraction [11, 12].

Researchers have begun utilizing mesoporous silica nanoparticles (MSN) due to their echogenic nature and ability to enhance the diagnostic capacity of ultrasound in disparate cancers [13, 14]. Furthermore, MSNs have a longer duration within circulation and are readily functionalizable allowing for the conjugation of various moieties $[15,16]$. In our previous work MSNs were functionalized with trastuzumab in order to provide a combined therapeutic and diagnostic agent for breast cancer treatment. The hybrid trastuzumab-MSN conjugates produced increased contrast ultrasound images with aggregation of the contrast around the HER2+ cancer cells [13]. However this work relied solely on the echogenic properties of the MSNs. In this work we propose the conjugation of a fluorocarbon (FC) to the surface of each MSN in order to further enhance the echogenicity and subsequent clinical potential. FCs have long been used as a reliable and stable ultrasound contrast agent and thus may provide a simple addition to increase the ultrasound sensitivity of the trastuzumab-MSN conjugates [17]. It has also been shown that the morphology of the nanoparticles can markedly alter their echogenicity [18, 19]. Although previously investigated tubular MSN have proven to increase therapeutic and diagnostic effects in breast cancer cells, alternative morphologies may produce greater results [20]. By comparing various MSN shapes (e.g. amorphous, spherical, and tubular) along with the conjugation of a FC, we hope to optimize both the delivery of trastuzumab and ultrasound contrast, while maximizing the diagnostic effects.

In this work, we propose a trastuzumab-MSNFC system that an improved diagnostic approach to HER2+ breast cancer. To accomplish this, we synthesized a previously described multifunctional, hybrid polymer-nanoparticle system using MSN, polyethylene glycol (PEG), and perfluorocarbon (Fig. 1). Fourier transform infrared spectroscopy (FT-IR) was used to confirm the successful conjugation of each component. Next each system was analyzed for increased ultrasound and compared to one another through mean pixel intensity (MPI). Finally, each system was conjugated to trastuzumab and exposed to HER2+ expressing breast cancer cells. Through this experiment we wished to analyze how the conjugation of FC as well as MSN shape would affect uptake of therapeutic moieties.

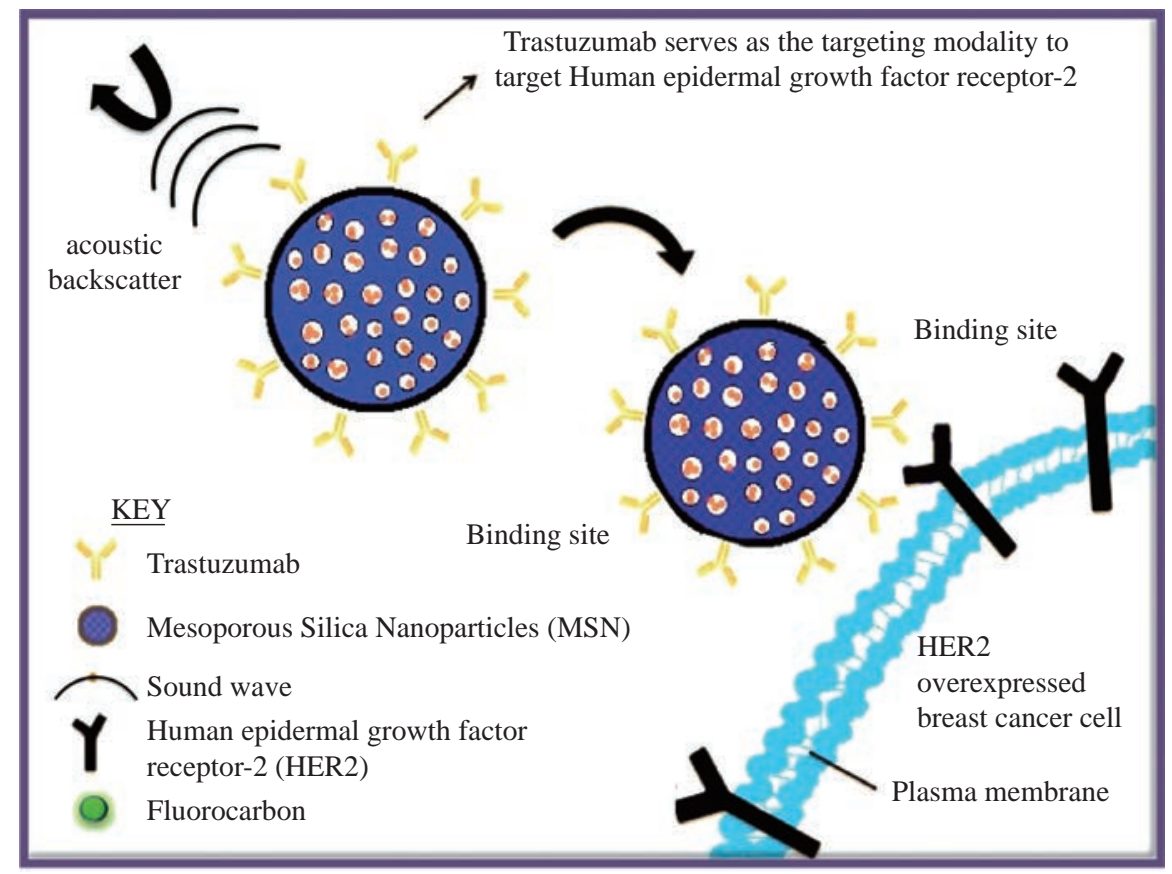

Fig. 1 Schematic of proposed mechanism of action of designed nanoparticle system. Mesoporous silica nanoparticles were conjugated with both trastuzumab and fluorocarbon to increase specificity of HER2 binding and ultrasound contrast respectively. 


\section{Experimental}

Detailed experimental methods are provided in the Supplementary Information (SI) Materials and Methods.

\section{Ultrasound apparatus}

Single-pulse ultrasound measurements were obtained using Non-Destructive Testing (NDT) with a frequency of $6.6 \mathrm{MHz}$ at a depth of $2.5 \mathrm{~cm}$. (GEPanametric). All transducers were Accuscan type $\mathrm{S}$ immersion transducers with point target focus (PTF). Receiver signal was acquired on an Inniium 8000 High Performance Oscilloscope (Agilent Technologies).

\section{Quantifying pixel intensity}

The main purpose of the ultrasound assay was to quantify the average pixel intensity for each of the synthesized nanoparticles, with and without the FC conjugation. The MSN of varying concentrations ( 0 , $0.5,1,2$, and $5 \mathrm{mg} / \mathrm{mL}$ ) were dispersed in $5 \mathrm{~mL}$ PBS. Each solution was sonicated for 10 minutes followed by placing the samples within dialysis tubing (Spectra/ Por 4, 25 mm, MWCO 12000-14000). Immediately following the addition of the samples, the dialysis tubings were then placed in a PBS bath on top of an agar gel. Agar served as a tissue-mimicking phantom and also served to reduce extraneous noise emitted into the dialysis tubing. The agar gels were made by autoclaving $3 \%$ agar in $\mathrm{dH}_{2} \mathrm{O}$ at $250{ }^{\circ} \mathrm{C}$ for 30 minutes. The liquid agar was then poured in plastic containers and allowed to cool, forming a gel. Ultrasound images were taken at room temperature. Three images were taken of each sample at each concentration and were analyzed using Image $\mathrm{J}$ to discover the mean pixel intensity. Each image was 150 x 100 pixel squares with an area of 150,000.

\section{Cell preparation}

HER2+ cells were prepared in a solution of DMEM F12 media with $10 \%$ fetal bovine serum (FBS) and $1 \%$ penicillin/streptomyocin. All cell assays were completed in triplicate. HER2+ cells were seeded at a density of 50,000 cells/well in 24 well plates.

\section{Fluorescent microscopy}

Six experimental samples were tested: AmorphousHerceptin, Sphere-Herceptin, Tubular-Herceptin, Amorphous-FC-Herceptin, Sphere-FC-Herceptin and Tubular-FC-Herceptin. All samples were analyzed at a concentration of $25 \mu \mathrm{g}$ nanoparticles/well for 30 minute and 2 hour incubation periods. Following the end of the incubation period, all samples were fixed using 10\% Neutral Buffered Formalin (NBF) for 15 minutes at room temperature, washed using PBS and stained with DAPI (Hoechst, 1:2000) for 10 minutes. Samples were imaged using a confocal microscope.

\section{Zeta-sizer}

Six experimental samples were tested: AmorphousHerceptin, Sphere-Herceptin, Tubular-Herceptin, and all unconjugated counterparts. Samples were dispersed in water and analyzed using a Zeta-sizer.

\section{Statsitics}

To analyze the zeta-sizer data for statistical significance a Kruskal-Wallis test was performed using GraphPad Prism 8.1.2.

\section{Results and Discussion}

Our goal in this work was the development of a nanoparticle system capable of increasing diagnostic imaging in breast cancer applications. In the subsequent sections we will discuss MSN synthesis and modification, utility as an ultrasound contrast agent, and the effects of trastuzumab conjugation on this system.

\section{Nanoparticle synthesis and characterization}

We began with the synthesis and characterization of the nanoparticle system. First MSNs were constructed to possess three different morphologies (e.g. amorphous, spherical, and tubular) as described in the methods section. Initial analysis of these MSN particles showed aggregation and decreased solubility, making them unsuitable as an injectable system [21]. For this reason PEG, a highly hydrophilic polymer, was conjugated to the surface of the MSNs to improve the dispersion of the nanoparticles (Fig. S1). Furthermore, the pegylation of the nanoparticles provided the additional benefit of preventing biomolecule adsorption onto the nanoparticle surface which can significantly change the characteristics of the nanoparticle [22, 23]. Pegylation works to prevent the formation of this protein layer and thus can preserve the integrity of the nanoparticle and prevent early clearance from circulation [23]. Next the pegylated MSNs were conjugated with FC to increase the ultrasound contrast of the nanoparticle. Typically nanoscale agents, such as MSNs, possess a relatively low ultrasound contrast performance. For this reason, higher doses of nanoscale 
contrast agents are required to obtain significant ultrasound enhancement, which can increase the risk of potential toxicity [24]. In order to decrease the required dose of MSNs while still achieving high diagnostic value, FCs, known to enhance imaging contrast, were conjugated to the surface of the nanoparticles. Next we attached the antibody trastuzumab to the MSN surface to provoke targeting of the MSN-FC system to HER2+ cancer cells. Trastuzumab was selected for this study as it has been shown to have significant clinical efficacy against HER2+ breast cancers, thereby providing a reliable moiety for MSN-FC conjugation [25].

The spectra after pegylation and FC conjugation can be viewed in Fig. 2. Each modification of the MSN was confirmed using FT-IR. The FT-IR spectrum of MSN alone was used as a baseline to confirm the subsequent conjugation of various moieties through the appearance of the appropriate peaks. Through the FT-IR spectra of solely MSNs shown in Fig. 2, we can see a peak at $1079-1088 \mathrm{~cm}^{-1}$ indicating the vibrations of Si-O-Si present within MSNs [26]. The second FT-IR spectrum shown in Fig. 2 (MSN-PEGHDI) was performed to show the successful pegylation of the MSNs; however, the peak intensities of the MSNs prove to be significantly higher than that of
PEG causing dominance of the MSN spectra that was seen [27]. Conjugation of PEG would typically be seen through the presence of hydroxyl groups in the region around $3378 \mathrm{~cm}^{-1}$ and $\mathrm{C}-\mathrm{O}$ stretching seen in the regions 1362 and $1287 \mathrm{~cm}^{-1}$ [28]. We confirmed the successful conjugation of FC to the MSNs by viewing the regions $1050-1132 \mathrm{~cm}^{-1}$, corresponding to C-F stretching [29] (Fig. 2).

It is well known that nanoparticle shape can have various effects on both the therapeutic and diagnostic potential of a system. Therapeutically, it has been reported that tubular or particles with a longer edge have a higher cellular uptake as compared with spherical or cuboidal counterparts [30-33]. Furthermore, tubular nanoparticles have been shown to increase systemic circulation time as compared to nanospheres [31]. Diagnostically, however; the size and shape of MSNs has yet to be optimized to enhance echogenicity [34, 35, 13]. In order to investigate how the size and shape of MSNs affects the nanoparticle's therapeutic and diagnostic capabilities, we first needed to synthesize and confirm each appropriate shape of nanoparticle (amorphous, spherical, and tubular). Transmission and scanning electron microscopy (TEM and SEM respectively) were used to investigate the morphology and shape of the MSNs and size

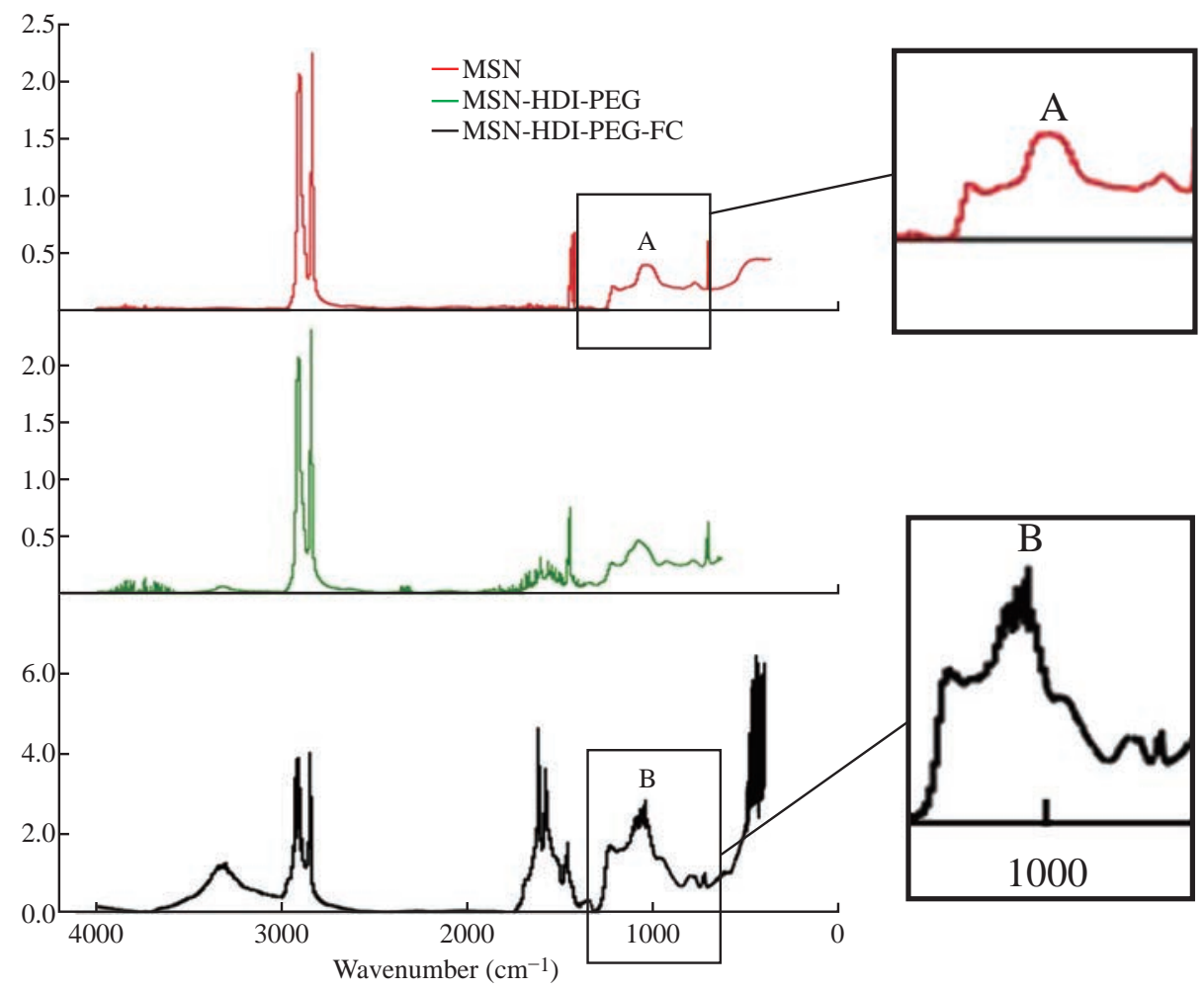

Fig. 2 Fourier transform infrared (FT-IR) spectroscopy was used to confirm the successful conjugation of each moiety to the surface of the MSNs. Peak A showing Si-O-Si vibrations at 1079-1088 $\mathrm{cm}^{-1}$. Peak B showing FC stretching at 1050-1132 $\mathrm{cm}^{-1}$. Peak C showing hydroxyl group vibrations at $3378 \mathrm{~cm}^{-1}$. 
distribution was determined through Zeta-Potential. Images of amorphous, spherical, and tubular shaped nanoparticles are displayed in Fig. 3. Fig. 3(a) and 3D show successful synthesis of amorphous nanoparticles with various sizes. Fig. 3(d) specifically reveals a much larger agglomerate of greater than 10 um. Fig. 3(b) and 3(e) depict successful synthesis of spherical nanoparticles ranging in 1-2 microns in diameter with good porosity, as indicated by the porous membrane. The SEM image (Fig. 3(e)) of the synthesized spherical nanoparticles displays some disparity in size of each particle. The tubular shaped nanoparticles can be seen in Fig. 3(c) and (f). Fig. 3 displays the average size, standard deviation, and polydispersity index (PDI) of each nanoparticle with and without Herceptin conjugation. Amorphous nanoparticles showed the largest PDI (0.78), followed by Herceptin conjugated amorphous nanoparticle (0.12). The wide range in size of unconjugated amorphous MSN may be due from the lack of definitive shape and possible clumping of the particles when recording. Spherical nanoparticles and Herceptin conjugated tubular nanoparticles showed PDIs of 0.09 and 0.08 respectively. Finally, tubular nanoparticles and Herceptin conjugated spherical nanoparticles showed the smallest PDI at 0.03 .

\section{Ultrasound Imaging}

Following SEM/TEM analysis, we investigated the three different MSN shapes (e.g. amorphous, spherical, and tubular) and their ability to serve as an ultrasound contrast agent. Subsequently, each particle shape was analyzed as an ultrasound contrast agent once conjugated with FC. Three images were obtained for each sample prepared and the mean pixel intensity (MPI) was obtained in ImageJ. The averaged MPI of the ultrasound images was then plotted (Fig. 4 and 5). MPI provides a global measure by averaging the pixel intensities across the image. On the other hand, integrated pixel intensity provides a sum of all pixels (each with its own intensity) in an image. Therefore, higher integrated pixel intensity can indicate a higher fluorescence per pixel or a lower fluorescence over a larger area. MPI was selected as the measure in this study as it is not affected by contrast concentration and provides a more standardized measure of contrast intensity. As shown in Fig. 4, there were differences in the MPI between the various shapes depending on the concentration of MSNs used. However, high doses of MSNs (above $1.0 \mathrm{mg} / \mathrm{mL}$ ) have been shown to generate reactive oxygen species (ROS). For this reason, we were interested in the difference between the MSN shapes at concentrations below this toxic dose. With this in mind, tubular MSNs were observed to produce the highest ultrasound contrast at these lower concentrations. Previous studies have shown that the shape of the nanoparticle can greatly impact
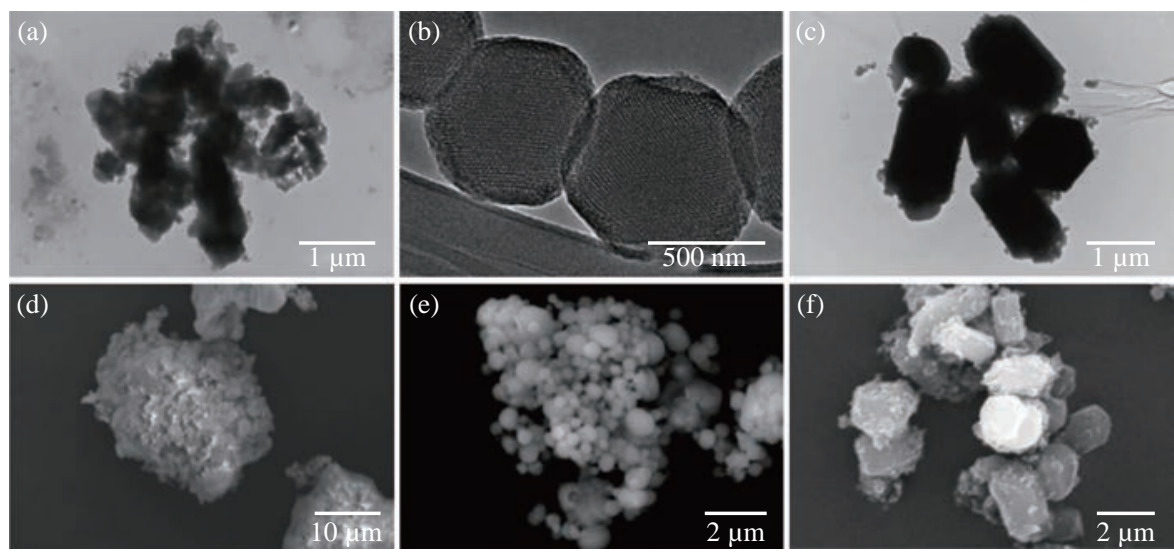

\begin{tabular}{|c|c|c|c|c|c|c|}
\hline & \multicolumn{6}{|c|}{ Zeta-Sizer Results (nm) } \\
\hline & $\begin{array}{c}\text { Tubular- } \\
\text { Herceptin }\end{array}$ & Tubular & $\begin{array}{c}\text { Spherical- } \\
\text { Herceptin }\end{array}$ & Spherical & $\begin{array}{c}\text { Amorphous- } \\
\text { Herceptin }\end{array}$ & Amorphous \\
\hline Average & 2032.7 & 1148.7 & 2124.0 & 1133.7 & 1155.0 & 2374.8 \\
\hline Std Dev & 568.4 & 207.9 & 369.7 & 331.6 & 401.7 & 2087.7 \\
\hline PDI & 0.08 & 0.03 & 0.03 & 0.09 & 0.12 & 0.78 \\
\hline
\end{tabular}

Fig. 3 Size distribution of the various MSNs. Electron microscopy images of MSN for structural characterization analysis. (a)-(c) TEM images of amorphous, spherical, and tubular MSNs respectively. (d)-(f) SEM images of amorphous, spherical, and tubular MSNs respectively. Average diameter, standard deviation, and PDI of MSNs through zeta-sizer. Please note varying scale bars for appropriate size approximation. 

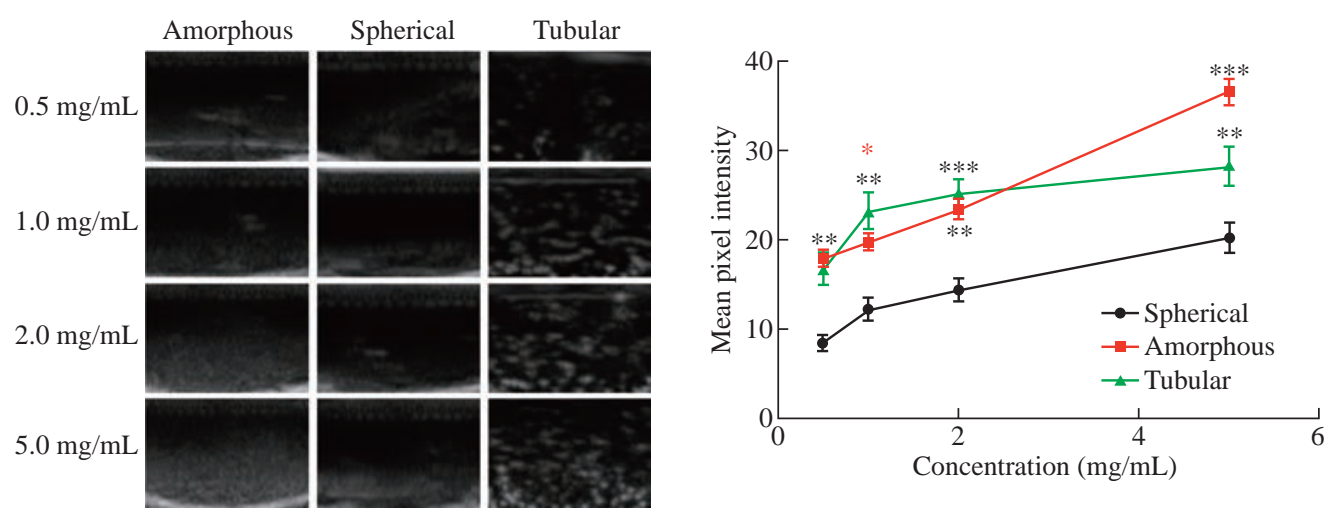

Fig. 4 Ultrasound images of each nanoparticle shape (amorphous, spherical, and tubular) at various concentrations $(0.5-5.0 \mathrm{mg} / \mathrm{mL})$ followed by analysis of mean pixel intensities of the ultrasound studies. * $\mathrm{p}<0.05,{ }^{* *} \mathrm{p}<0.01,{ }^{* * *} \mathrm{p}<0.001$.
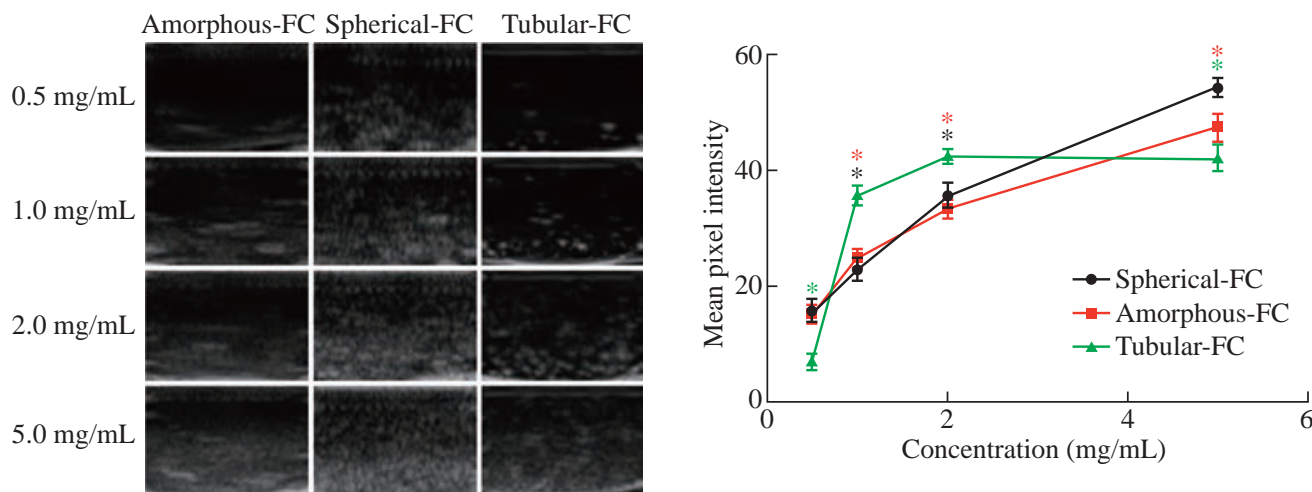

Fig. 5 Ultrasound images of each nanoparticle shape (amorphous, spherical, and tubular) conjugated with fluorocarbon (FC) at various concentrations $(0.5-5.0 \mathrm{mg} / \mathrm{mL})$ followed by analysis of mean pixel intensities of the ultrasound studies. ${ }^{*} \mathrm{p}<0.05$.

the cellular uptake, affinity to blood proteins, and circulation time [36]. However, it has also been shown that the shape of nanoparticle can have considerable effects on the absorption of imaging waves thus impacting contrast capacity [37]. It is important to note that MSN contrast can also vary depending on the size of the particle. Thus, it is possible that larger spherical MSNs could provide increased ultrasound contrast, more similar to that of the tubular MSNs [34]. Furthermore, images provided in Fig. 4 and 5 represent integrated pixel intensity by showing the total intensity across the entire field; however, as mentioned before these images are affected by concentration. We next looked at the effects of FC conjugation to the various shaped MSNs (Fig. 5). FC conjugation was performed to increase the robust diagnostic capacity of the MSNs. In addition, covalent conjugation of the FCs to the MSNs compared to simple encapsulation eliminates the possibility of these moieties spreading beyond the vicinity of the nanoparticles. It was determined that the conjugation of the FC increased the echogenic properties of the MSN at most concentrations, regardless of shape. The increase of concentration of the MSNs was also found to increase the MPI consistent among all shapes with and without the FC conjugation. Together these results indicate that tubular shaped nanoparticles conjugated with FC may provide the greatest ultrasound contrast at lower concentrations making them more suited for ultrasound applications.

\section{In-vitro studies}

It has previously been shown that the shape of a nanoparticle can affect how these efficiently these particles traverse the different cellular barriers and are taken up by cells. In fact, previous research has shown that high aspect ratio nanoparticles (i.e. rods and tubes) have a higher ratio of cellular uptake than those with a lower aspect ratio (i.e. spheres) [38-40]. However it is important to note that the type of cell, material composition of the nanoparticle, and size of the nanoparticle can also play a significant role in the rate of nanoparticle ingestion [41, 42]. To understand how the shape of MSNs affects cellular uptake, each of the MSN shapes were incubated for either 30 minutes or 2 hours with HER2 overexpressing breast cancer cells. As these particles are fluorescently labeled, we were able to witness the amount of cellular uptake by 
tracking the fluorescence. Fig. 6 displays the images of the various cell cultures incubated with MSNs obtained through fluorescent microscopy. All nanoparticles were conjugated with a fluorescent label, FITC, and all cells underwent a nuclei stain. Images were analyzed qualitatively for co-localization of nanoparticles to HER2 overexpressing cells. We observed that colocalization was found following both the 30 minute and 2 hour incubation periods; however, a greater amount of nanoparticles were found to co-localize to the HER2+ cells at the 2 hour incubation period as compared to the 30 minute incubation period. This is likely because more time allowed for more cells to endocytose the MSNs.

In addition, FC conjugation to the surface of the nanoparticles did not seem to interfere with the successful binding of Herceptin to the HER2+ receptor as co-localization of the cells and fluorescently labeled particles were found in all FC groups. Furthermore, the FC-Herceptin particles displayed an equal or greater amount of co-localization with the cells as compared to their non-FC counterpart. To analyze these results, images were taken for each of the six experimental samples. Image $\mathrm{J}$ was then used to quantify the percent area of nanoparticles per frame through quantifying the level of FITC expression per frame. Table 1 displays the percent of the area of nanoparticles relative to frame of image of each sample group. The percentage of MSNs per frame increased when the incubation time increased from 30 minutes to 2 hours. Additionally the conjugation of a FC increased the amount of colocalization of the MSNs to the HER2+ cells. This further indicates that the FC does not interfere with the interaction of Herceptin and the HER2+ receptor. The sample with the highest amount of FITC expression per frame was determined to be Tubular-FC-Herceptin at the 2-hour incubation period. These fluorescent images may suggest that the tubular shaped morphology has a higher rate of cellular endocytosis when compared to the hexagonal or spherical particles. It is important to note that cellular endocytosis can cause cellular lysis of the target cells [43]; however, additional cell studies using a live/dead assay would need to be performed in order to confirm cell lysing due to the endocytosis of the nanoparticles. It is important to note that the exact effects of the FC on the MSN properties were not obtained due to a lack of a FC control. Therefore, one limitation is the variations in results following FC conjugation that could be a result of the conjugation itself or its effects on the size/shape of the nanoparticle.

The design of this study was conducted in order to enhance the diagnostic effects of mesoporous nanoparticles for the targeted diagnosis of breast cancer. Modalities are quickly progressing into becoming

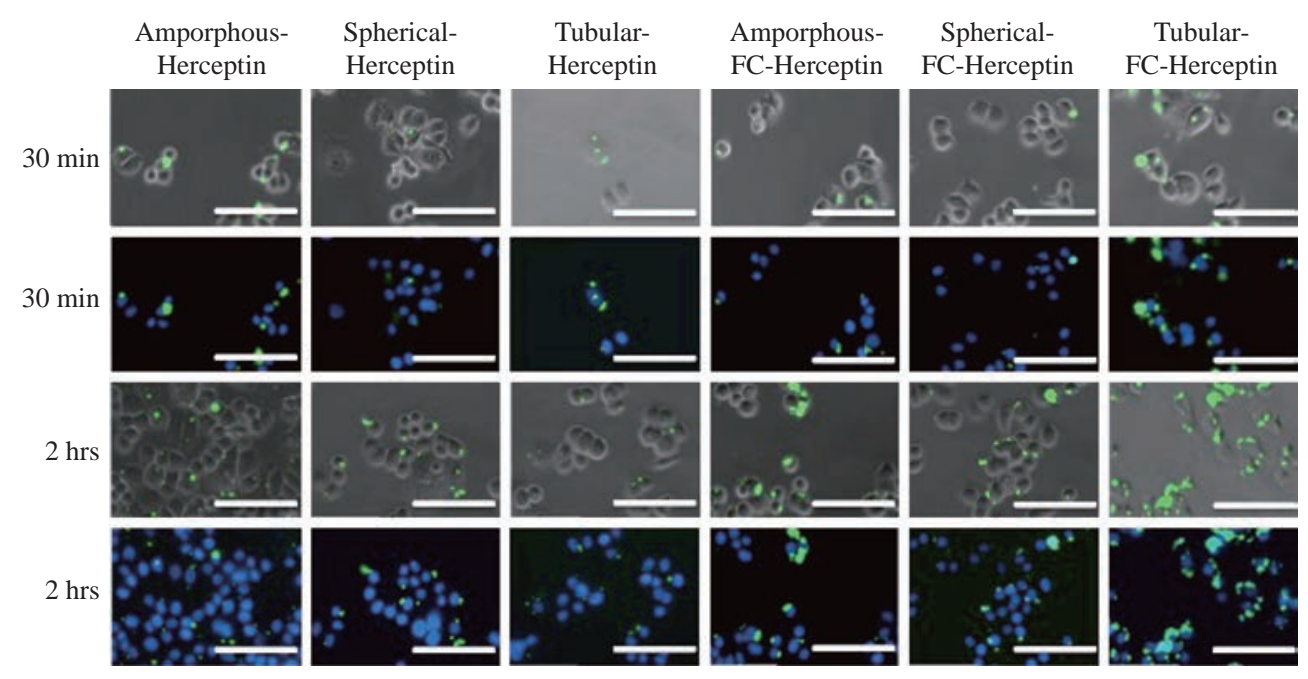

Fig. 6 Fluorescent images of MSNs with and without FC attachment incubated with HER2+ cells for 30 minutes or 2 hours. Green staining indicates the presence of FC conjugated MSNs. Blue staining indicates DAPI staining. Images were taken at 40×. Scale bar is $100 \mu \mathrm{m}$.

Table 1 The percent of the area from the particles: Frame of image was found utilizing Image $\mathrm{J}$ and is reported. 10× magnification images were utilized to obtain the percent area of particles

\begin{tabular}{ccccccc}
\hline Incubation time & Amorphous & Spherical & Tubular & Amorphous-FC & Spherical-FC & Tubular-FC \\
\hline 30 minutes & 0.148 & 0.009 & 0.006 & 0.314 & 0.043 & 0.203 \\
2 hours & 0.311 & 0.357 & 0.165 & 0.46 & 0.332 & 3.046 \\
\hline
\end{tabular}


multifunctional, serving to enhance diagnostic imagery as well as offer means of therapeutic delivery or attachment of therapeutic moieties. Ultrasound is a cost-effective, non-ionizing and bedside friendly imaging system as compared to the current clinical standard, mammography, for diagnosing and tracking the progression of the tumor [7]. There are several ultrasound contrast agents utilized in clinical practice including gas microbubbles and nanoparticles [44, 45]. However, gas microbubbles have an extremely short lifespan within systemic circulation, lasting only a few minutes [46]. To circumvent these shortcomings, we analyzed the ability of FC-conjugated MSNs in various morphologies to enhance diagnostic effects.

In this work three morphologies of MSNs were tested to determine their ultrasound contrast at various concentrations along with their FC conjugated counterpart. A general trend was seen which depicted the FC conjugated particles emitted a higher MPI as compared to the non-FC conjugated MSNs. Tube and amorphous MSNs alone were shown to have a higher contrast than the spherical MSNs at all concentrations and tube MSNs appeared to have higher contrast than amorphous at lower concentrations. To test the targeted MSN's capability of being endocytosed to their targeted cells, an in vitro assay was performed. The MSN-FC-HER particles were reported to have a higher percent area of particles to image frame when compared to the non-FC conjugated MSNs. Future studies aim at establishing therapeutic benefit of MSNFC-HER through target binding assays with HER2 positive breast cancer cells. Additional studies will also include more in-depth characterization of the surface chemistry and kinetic properties of the MSNs with and without the various surface conjugations. These characteristics can have tremendous effects on the absorption, distribution, binding, and excretion of nanoparticles and may provide us with additional features to tailor and further improve their diagnostic and therapeutic capacity $[47,48]$. Furthermore, key studies will be required to determine the behavior and efficacy of these nanoparticles following clinical delivery, similar to the currently used imaging enhancing modalities.

\section{Conclusions}

In summary, a multifunctional, hybrid nanoparticle system was successfully developed to enhance contrast in ultrasound for application in breast cancer diagnostics. Three morphologies were tested in their capability for ultrasound contrast and rate of endocytosis. It can be suggested that tubular MSNs emit a higher contrast alone and all morphologies share a general trend of increased contrast with the conjugation of a FC. Overall, these results suggest that MSNs may be designed to possess the ideal size, shape, and chemical modification to further enhance contrast intensity in ultrasound. We hope that this research can provide a platform for future research in improving the ability of ultrasound to diagnosis and monitor breast cancers.

\section{Acknowledgements}

The electron microscopy lab at Children's Hospital Colorado provided advice and guidance related to the SEM/TEM that was critical to this project.

\section{Author Contributions}

Melissa Ronni Laughter performed data analysis and manuscript writing. Anna Laura Nelson performed data collection and data analysis. Maria Bortot performed data analysis. Brisa Pena provided direction during the study and aided in imaging. Bolin Liu provided direction during the study and aided in cellular studies. Daewon Park conceived the study and provided the means and direction for the synthesis and characterization of this MSN system.

\section{Funding}

This work was supported by University of Colorado Denver Start-up funding for Dr. Park.

\section{Conflict of Interests}

The authors declare that they have no conflict of interest.

\section{References}

[1] R.L. Siegel, K.D. Miller, and A. Jemal, Cancer statistics, 2018. CA. Cancer J. Clin. [Internet], 2018, 68(1): 7-30.

[2] M.M. Moasser, Targeting the function of the HER2 oncogene in human cancer therapeutics. Oncogene, 2011, 26(46): 6577-6592.

[3] G. Demonty, C. Bernard-Marty, F. Puglisi, et al., Progress and new standard (1); G. Demonty, C. Bernard-Marty, F. Puglisi, et al., Progress and New standards of care in 
the management of HER-2 positive breast cancer. Eur. $J$. Cancer, 2007, 43(3): 497-509.

[4] E.H. Romond, E.A. Perez, J. Bryant, et al., Trastuzumab plus adjuvant chemotherapy for operable HER2-positive breast cancer. N Engl J Med, 2005; 353(16): 1673-1684.

[5] M. Khairalseed, K. Javed, G. Jashkaran, et al., Monitoring early breast cancer response to neoadjuvant therapy using h-scan ultrasound imaging. J. Ultrasound Med, 2019; 38(5): 1259-1268.

[6] J. Fernandes, L. Sannachi, W.T. Tran, et al., Monitoring breast cancer response to neoadjuvant chemotherapy using ultrasound strain elastography. Transl. Oncol, 2019, 12(9): 1177-1184.

[7] R. Guo, G. Lu, B. Qin, and B. Fei, Ultrasound Imaging Technologies for Breast Cancer Detection and Management: A Review. Ultrasound Med. Biol. [Internet], 2018, 44(1): 37-70.

[8] J. Geisel, M. Raghu, and R. Hooley, The role of ultrasound in breast cancer screening: The case for and against ultrasound. semin. Ultrasound, CT MRI, 2018, 39(1): 25-34.

[9] M.A. Hahn, A.K. Singh, P. Sharma, et al., Nanoparticles as contrast agents for in-vivo bioimaging: Current status and future perspectives. Anal. Bioanal. Chem, 2011, 399(1): 3-27.

[10] M. Figueiredo, R. Esenaliev, PLGA nanoparticles for ultrasound-mediated gene delivery to solid tumors. $J$. Drug Deliv, 2012, 2012: 767839.

[11] X. Wei, Y. Li, S. Zhang, X. Gao, et al., Ultrasound targeted apoptosis imaging in monitoring early tumor response of trastuzumab in a murine tumor xenograft model of Her-2-positive breast cancer. Transl. Oncol, 2014, 7(2): 284-291.

[12] K.A. Kaphingst, S. Persky, and C. Lachance, Theranostic imaging of cancer. Eur. J. Radiol, 2012, 14(4): 384-399.

[13] A. Milgroom, M. Intrator, K. Madhavana, et al., Mesoporous silica nanoparticles as a breast-cancer targeting ultrasound contrast agent. Colloids Surf B Biointerfaces, 2016, 116: 652-657.

[14] A.A. Alexander-Bryant, W.S. Vanden Berg-Foels, and $\mathrm{X}$. Wen, Bioengineering strategies for designing targeted cancer therapies. Bioengineering, 2014, 118: 1-59.

[15] S. Kwon, R.K. Singh, R.A. Perez, et al., Silica-based mesoporous nanoparticles for controlled drug delivery. $J$. Tissue Eng, 2013, 4: 20-22.

[16] L.B. Peppas, Recent advances on the use of biodegradable microparticles and nanoparticles in controlled drugdelivery. Int. J. Pharm, 1995, 116(1): 1-9.

[17] J.M. Correas, L. Bridal, A. Lesavre, et al., Ultrasound contrast agents: Properties, principles of action, tolerance, and artifacts. Eur. Radiol, 2001, 11(8): 1316-1328.

[18] Y.J. Wong, L. Zhu, W.S. Teo, et al., Revisiting the Stöber method: Inhomogeneity in silica shells. J. Am. Chem. Soc, 2011, 133(30): 11422-11425.

[19] K. Zhang, H. Chen, X. Guo, et al., Double-scattering/ reflection in a single nanoparticle for intensified ultrasound imaging. Sci. Rep, 2015, 5: 1-11.

[20] X. Huang, L. Li, T. Liu, et al., The shape effect of mesoporous silica nanoparticles on biodistribution, clearance, and biocompatibility in vivo. ACS Nano, 2011, 5(7): 5390-5399.

[21] B.G. Amsden, Liquid, injectable, hydrophobic and biodegradable polymers as drug delivery vehicles. Macromol. Biosci, 2010, 10(8): 825-835.

[22] V. Cauda, A. Schlossbauer, and T. Bein, Bio-degradation study of colloidal mesoporous silica nanoparticles: Effect of surface functionalization with organo-silanes and poly(ethylene glycol). Microporous Mesoporous Mater, 2010, 132(1-2): 60-71.

[23] J.Y. Oh, H.S. Kim, L. Palanikumar, et al., Cloaking nanoparticles with protein corona shield for targeted drug delivery. Nat. Commun, 2018, 9: 4548.

[24] A. Yildirim, R. Chattaraj, N.T. Blum, et al., Stable encapsulation of air in mesoporous silica nanoparticles: fluorocarbon-free nanoscale ultrasound contrast agents. Adv. Healthc. Mater, 2016, 5(11): 1290-1298.

[25] K. Mann, M. Kullberg, Trastuzumab-targeted gene delivery to Her2-overexpressing breast cancer cells. Cancer Gene Ther, 2016, 23(7): 221-228.

[26] H. Sanaeishoar, M. Sabbaghan, and F. Mohave, Synthesis and characterization of micro-mesoporous MCM-41 using various ionic liquids as co-templates. Microporous Mesoporous Mater, 2015, 217: 219-224.

[27] A. Dabbagh, R. Mahmoodian, B.J.J. Abdullah, et al., Low-melting-point polymeric nanoshells for thermaltriggered drug release under hyperthermia condition. Int. J. Hyperth, 2015, 31(8): 920-929.

[28] L.E. van Vlerken, M.M. Amiji, Multi-functional polymeric nanoparticles for tumour-targeted drug delivery. Expert Opin. Drug Deliv, 2006, 3(2): 205-216.

[29] S. Sahoo, C.K. Chakraborti, and P.K. Behera, FTIR and Raman spectroscopic investigations of Ofloxacin / Carbopol 940 mucoadhesive suspension. Int. J. PharmTech Res, 2012, 4(1): 382-391.

[30] S.E.A. Gratton, P.A. Ropp, P.D. Pohlhaus, et al., The effect of particle design on cellular internalization pathways. Proc. Natl. Acad. Sci, 2008, 105(33): 1161311618.

[31] A. Wani, G.H.L. Savithra, A. Abyad, et al., Surface PEGylation of mesoporous silica nanorods (MSNR): Effect on loading, release, and delivery of mitoxantrone in hypoxic cancer cells. Sci. Rep, 2017, 7(1): 1-11.

[32] X. Huang, X. Teng, D. Chen, et al., The effect of the shape of mesoporous silica nanoparticles on cellular uptake and cell function. Biomaterials, 2010, 31(3): 438448.

[33] A. Banerjee, J. Qi, R. Gogoi, et al., Role of nanoparticle size, shape and surface chemistry in oral drug delivery HHS public access. J Control Release, 2016, 238(805): 176-185.

[34] S. Casciaro, F. Conversano, A. Ragusa, et al., Optimal enhancement configuration of silica nanoparticles for ultrasound imaging and automatic detection at conventional diagnostic frequencies. Invest. Radiol, 2010, 45(11): 715-724.

[35] P.J. Kempen, S. Greasley, K.A. Parker, et al., Theranostic mesoporous silica nanoparticles biodegrade after prosurvival drug delivery and ultrasound / magnetic resonance imaging of stem cells. Theranostics, 2015, 5(6): 631-642.

[36] A. Chonn, S.C. Semple, and P.R. Cullis, Association of blood proteins with large unilamellar liposomes in vivo. Relation to circulation lifetimes. J. Biol. Chem, 1992, 267(26): 18759-18765.

[37] P. Jackson, S. Periasamy, V. Bansal, et al., Evaluation of the effects of gold nanoparticle shape and size on contrast enhancement in radiological imaging. Australas. Phys. Eng. Sci. Med, 2011, 34(2): 243-249.

[38] V.T. Cong, K. Gaus, R.D. Tilley, et al., Rod-shaped mesoporous silica nanoparticles for nanomedicine: recent progress and perspectives. Expert Opin. Drug Deliv, 2018, 15(9): 881-892.

[39] N.P. Truong, M.R. Whittaker, C.W. Mak, et al., The importance of nanoparticle shape in cancer drug delivery. Expert Opin. Drug Deliv, 2015, 12(1): 129-42.

[40] C. Kinnear, T.L. Moore, L. Rodriguez-Lorenzo, et al., Form follows function: nanoparticle shape and its implications for nanomedicine. Chem. Rev, 2017, 117(17): 11476-11521.

[41] K. Kettler, K. Veltman, D. van de Meent, et al., Cellular 
uptake of nanoparticles as determined by particle properties, experimental conditions, and cell type. Environ. Toxicol. Chem, 2014, 33(3): 481-492.

[42] F. Lu, S.H. Wu, Y. Hung, et al., Size effect on cell uptake in well-suspended, uniform mesoporous silica nanoparticles. Small, 2009, 5(12): 1408-1413.

[43] Q. Mu, N.S. Hondow, L. Krzemiński, et al., Mechanism of cellular uptake of genotoxic silica nanoparticles. Part. Fibre Toxicol, 2012, 9: 1-11.

[44] E. Quaia. Microbubble ultrasound contrast agents: An update. Eur. Radiol, 2007, 17(8): 1995-2008.

[45] D. Cosgrove. Ultrasound contrast agents: An overview. Eur. J. Radiol, 2006, 60(3): 324-330.

[46] F. Calliada, R. Campani, O. Bottinelli, et al., Ultrasound contrast agents: Basic principles. Eur. J. Radiol, 1998, 27: 157-160.
[47] A. Albanese, P.S. Tang, and W.C.W. Chan, The effect of nanoparticle size, shape, and surface chemistry on biological systems. Annu. Rev. Biomed. Eng, 2012, 14 1-16.

[48] W.I. Hagens, A.G. Oomen, W.H. de Jong, et al., What do we (need to) know about the kinetic properties of nanoparticles in the body? Regul. Toxicol. Pharmacol, 2007, 49(3): 217-29.

Copyright $₫$ Melissa Ronni Laughter, Anna Laura Nelson, Maria Bortot, Brisa Pena, Bolin Liu, and Daewon Park. This is an open-access article distributed under the terms of the Creative Commons Attribution License, which permits unrestricted use, distribution, and reproduction in any medium, provided the original author and source are credited. 